\title{
Annual Conference of the Canadian Association of Professional Academic Librarians (CAPAL) - Congress 2014
}

The Canadian Association of Professional Academic Librarians / L'Association canadienne des bibliothécaires académiques professionnels (CAPAL/ACBAP) will hold its inaugural conference at the Congress of the Humanities and Social Sciences at Brock University from May 25-26, 2014. CAPAL will be joining over 70 scholarly associations to share ideas, knowledge, research and experience under the umbrella theme of "Borders Without Boundaries." The specific theme for CAPAL's conference, "Shifting Landscapes: Exploring the Boundaries of Academic Librarianship," speaks to a desire to bring together challenging perspectives relating to academic librarians while acknowledging the core values of academic librarianship. We aim to create a program that will challenge current thinking about professional issues, promote the exchange of ideas and enhance communication among our members, forge new relationships with other organizations that share our goals and values, and provide a venue to present research and scholarship.

We look forward to seeing you at the Congress in 2014. (http://congress2014.ca/)

Programme Planning Committee: Juliya Borie, Jennifer Dekker, Dean Giustini, Dave Hudson, Leona Jacobs, Rhiannon Jones, Mary Kandiuk, Harriet Sonne de Torrens, Mark Weiler

http://capalibrarians.org/

Le colloque inaugural de l'Association canadienne des bibliothécaires académiques professionnels (CAPAL/ACBAP) se tiendra lors du Congrès des sciences humaines à l'Université Brock les 25 et 26 mai 2014. CAPAL/ACBAP se joindra à plus de 70 associations académiques pour partager idées, connaissances, travaux de recherche et expériences sur le thème de «Frontières sans limites ». Le thème spécifique du colloque de CAPAL/ACBAP, «Environnements en constante transformation : exploration des limites de la bibliothéconomie académique», s'adresse à la volonté de rassembler les perspectives différentes liées aux bibliothécaires académiques tout en reconnaissant les valeurs fondamentales de la bibliothéconomie académique. Nous visons à créer un programme qui remettra en question les idées actuelles sur des sujets professionnels, promouvra l'échange des idées, offrira un endroit pour présenter les travaux de recherche et améliorera la communication entre nos membres tout en forgeant de nouvelles relations avec d'autres organisations qui partagent nos objectifs et nos valeurs.

Nous vous attendons avec impatience au Congrès de 2014. (http://congress2014.ca/)

Les membres du comité du programme : Juliya Borie, Jennifer Dekker, Dean Giustini, Dave Hudson, Leona Jacobs, Rhiannon Jones, Mary Kandiuk, Harriet Sonne de Torrens, Mark Weiler 